\title{
Factors associated with discussion of sexual activity and contraception in women with HIV
}

\author{
Zoe A Stewart, ${ }^{1}$ Kate Shipley, ${ }^{2}$ Tim Spelman, ${ }^{3}$ Michelle L Giles ${ }^{4,5,6}$
}

\begin{abstract}
${ }^{1}$ Medical Student, Monash Infectious Diseases, Monash University, Clayton, Victoria, Australia

${ }^{2}$ HIV Nurse, Infectious Diseases Unit, Alfred Health, Prahran, Victoria and Monash Infectious Diseases, Monash Health, Clayton, Victoria, Australia ${ }^{3}$ Biostatistician, Infectious Diseases Unit, Alfred Health, Prahran, Victoria, Australia ${ }^{4}$ Infectious Diseases Physician; Director, Infections in Pregnancy Service, Monash Infectious Diseases, Monash Health, Clayton, Victoria, Australia ${ }^{5}$ Deputy Director, Monash HIV, Monash Infectious Diseases, Monash Health, Clayton, Victoria, Australia

${ }^{6}$ Adjuct Clinical Associate Professor, Department of Obstetrics and Gynaecology, Monash University, Clayton, Victoria, Australia
\end{abstract}

\section{Correspondence to} Associate Professor Michelle Giles, Infectious Diseases Unit, Alfred Health, 2nd Floor, Burnet Institute, 85 Commercial Road, Prahran, Victoria 3181, Australia; m.giles@alfred.org.au

Received 20 March 2014 Revised 30 January 2015 Accepted 16 February 2015 Published Online First 21 April 2015

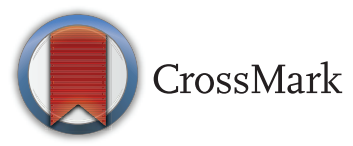

To cite: Stewart ZA, Shipley K, Spelman T, et al. $J$ Fam Plann Reprod Health Care 2016:42:12-16.

\begin{abstract}
Objectives Contraceptive use is important for reproductive-aged women living with HIV to plan and optimise safety of pregnancies. Clinicians play a vital role in counselling patients about contraception and safe sexual activity. This study aimed to determine the frequency and predictors of discussions regarding sexual activity and contraceptive use
\end{abstract}

Methods Retrospective clinical study of all reproductive-aged women $(n=128)$ treated for HIV between 2010 and 2012 at two metropolitan hospitals. Evidence of discussion between treating doctors and patients regarding sexual activity and contraception and variables including patient age, gender of doctor, time since HIV diagnosis, previous pregnancy, country of birth and antiretroviral regimen were obtained from medical records.

Results Sexual activity status was documented for $54 \%$ of the women and discussion regarding contraception was recorded for less than onethird of the study participants. Contraceptive use was not recorded in $63 \%$ of the medical records. In the study cohort $27 \%$ of the women used contraception, $10 \%$ did not use contraception, and when a discussion regarding sexual activity was documented, contraception was 3.7 times more likely to also be discussed ( $p=0.04$ ).

Excluding women who were pregnant, women who were documented as using contraception were 1.8 times as likely to have had a discussion about contraception documented $(p=0.05)$. After adjustment for other factors, previous pregnancy, gender of doctor and age of patient were not associated with discussions regarding sexual activity or contraception.

Conclusions Discussions regarding sexual activity and contraception between HIV-infected women of reproductive age and their clinicians were inconsistent and suboptimal. Mechanisms to facilitate regular discussion about sexual activity and contraception between clinicians and women with HIV warrant further investigation.

\section{Key message points}

- The rate of unintended pregnancy is high in women living with HIV.

- Discussions regarding sexual activity and contraception are important to prevent viral transmission and unwanted pregnancy.

- Documentation of these discussions remains inconsistent and suboptimal.

- Further research should examine mechanisms to ensure these conversations become a routine and regular part of caring for women living with HIV.

\section{INTRODUCTION}

Worldwide, the number of women infected with HIV continues to increase. Rates of unintended pregnancy are as high as 50-84\% among women living with $\mathrm{HIV}^{1} 2$ and contraceptive use is important to plan and optimise safety of pregnancy as well as prevent viral transmission. Barrier methods of contraception offer the benefit of preventing both unwanted pregnancy and viral transmission. Historically, there has been concern over the interaction of liver enzyme-inducing antiretroviral (ARV) therapy [particularly protease inhibitors (PIs) and non-nucleoside reversetranscriptase inhibitors (NNRTIs)] and hormonal contraceptives, with some studies finding an increased rate of HIV transmission. However, the relationship remains controversial and unclear. ${ }^{3-5}$ There is no evidence of association between hormonal contraceptives and disease progression for women living with HIV. $^{6}$

Published recommendations advise that sexual history should be documented every 6-12 months or as relevant, and that counselling regarding contraception 
should occur routinely and regularly for HIV-positive women. $^{78}$

The literature demonstrates that the decisions that women living with HIV make regarding contraceptives are often unrelated to the virus. ${ }^{9}$ These decisions, and the likelihood of compliance with the chosen method, are often multifaceted and are affected by socioeconomic status, partnership characteristics, past contraceptive use and pregnancy experience and attitudes. ${ }^{10}$ As with all populations, pre-pregnancy counselling in women living with HIV is variable, but evidence suggests that HIV-positive women and serodiscordant couples are open to discussions regarding pregnancy and contraception. ${ }^{11}$

This article examines the frequency and predictors of discussions regarding sexual activity, relationship status and contraception between clinicians and reproductive-aged women with HIV, and reviews rates of contraceptive use in these women.

\section{METHODS}

A retrospective clinical study was conducted of all reproductive-aged women treated for HIV between 2010 and 2012 at two metropolitan centres in Melbourne, Australia - the Alfred Hospital and Monash Medical Centre. Both centres are adult tertiary referral teaching hospitals, each with more than 500 inpatient beds and in excess of 100000 patients seen annually in specialist outpatient settings. ${ }^{12}$ Ethical approval for this study was obtained from The Alfred Health Ethics Committee.

Reproductive age was defined as $16-49$ years.

Electronic records of all HIV-related nursing and medical appointments were examined for evidence of discussions between women and their clinicians relating to relationship status, sexual activity, contraception and pregnancy. Demographic data were obtained from the Victorian HIV Service Database (VHIVSD). Hormonal contraceptive use and ARV regimens were obtained from medical and pharmacy records. Women were considered to have a 'regular clinician' if they saw the same physician for more than $50 \%$ of clinic visits. The clinicians at these two hospitals are predominantly infectious diseases physicians, not sexual health practitioners.

\section{Victorian HIV Service Database}

The VHIVSD contains records of all individuals treated for HIV infection at the Alfred Hospital (Melbourne, Australia) and the former Fairfield Infectious Diseases Hospital (Melbourne, Australia, closed in 1996). From 1996, data on current patients of the HIV Service have been entered prospectively, patients active prior to this date have had data entered retrospectively.

The VHIVSD stores records of HIV disease parameters such as HIV viral load, CD4 cell count and ARV therapy. In addition, it contains records of serological testing for other infections of interest including sexually transmitted infections (STIs). The same data were obtained from the Monash Infectious Diseases Service HIV database, which is modelled on the VHIVSD.

Unadjusted and adjusted Poisson regression modelling with robust standard errors (SEs) was performed to investigate predictors of evidence of discussion regarding contraception or sexual activity. Variables assessed include age of patient, gender of doctor, time since diagnosis of HIV, previous pregnancy, country of birth and ARV regimen. Candidate predictors for inclusion in each adjusted model were identified based on univariate modelling performance in addition to consideration of a priori known or suspected confounders.

Modified Poisson regression modelling with robust SEs was used to investigate predictors of evidence of discussion regarding contraception or sexual activity. A sandwich estimation robust error variance procedure was used to avoid overestimation of effect size secondary to the relative frequency of our outcome variables.

\section{RESULTS}

The medical records of 128 women were examined across the two centres (Centre 1: $n=87,68 \%$; Centre $2: n=41,32 \%)$. One woman was excluded from analysis because she was a postoperative male-to-female transgender person. The mean age of women was 36 (range 17-48) years and mean time since diagnosis was 9 (range 0-30) years. The majority of women were born outside Australia or New Zealand $(n=77 / 128$; $60 \%)$, predominantly in South-East Asia (29/128; $23 \%)$ and Africa $(34 / 128 ; 27 \%)$. Eight women were pregnant at some point during the study period and $30(23 \%)$ women had been pregnant previously. There were no differences in demographics or results between the two centres.

The majority of women were on ARV therapy (55/ $128,43 \%$ on PIs; $40 / 128,31 \%$ on NNRTIs; $3 / 128$, $2 \%$ on integrase inhibitors; $9 / 128,7 \%$ on other ARV therapy; $21 / 128,16 \%$ on no ARV therapy).

Documentation of discussion regarding contraception occurred in $28 \%$ of HIV-positive women of reproductive age (Table 1$)$. Sexual activity status and relationship status was documented in 54\% and $76 \%$ of cases, respectively. Of the 34 (27\%) women who were documented as using contraception, 20 women used condoms and 14 women used hormonal contraceptives (including oral contraceptive pills and hormonal long-acting reversible contraceptives; Table 2).

Of the 94 women who either did not use contraception or for whom contraceptive use was not documented, 11 were considering pregnancy or actively trying to conceive.

If sexual activity was documented, contraception was 3.7 times more likely to also be discussed $(p=0.04)$, after adjustment for other factors. Women 
Table 1 Documentation of discussion regarding contraception and sexual activity

\begin{tabular}{lrl}
\hline Scenario & $\begin{array}{l}\text { Yes } \\
{[\boldsymbol{n}(\%)]}\end{array}$ & $\begin{array}{l}\text { Not documented } \\
{[\boldsymbol{n}(\%)]}\end{array}$ \\
\hline Patient has a regular doctor & $112(88)$ & - \\
Patient is in a relationship & $77(60)$ & $31(24)$ \\
$\begin{array}{l}\text { Documented discussion about sexual } \\
\text { activity status }\end{array}$ & $69(54)$ & - \\
$\begin{array}{l}\text { Documented discussion about } \\
\text { contraception }\end{array}$ & $36(28)$ & - \\
$\begin{array}{l}\text { Documented that patient uses } \\
\text { contraception }\end{array}$ & $34(27)$ & $81(63)$ \\
\hline
\end{tabular}

who had a documented conversation about contraception with their clinician were almost twice as likely to actually be using contraception $(p=0.05)$ (Table 3 ).

On adjusted multivariate analysis there was no association between discussion regarding sexual activity or contraception and previous pregnancy, gender of doctor, age of patient, country of birth, time since diagnosis or ARV regimen after adjustment for all other covariates (Table 3 ).

\section{DISCUSSION}

This study demonstrated that clinician-patient discussions regarding sexual activity and contraception were inconsistent and suboptimal. Less than one-third of patients had a documented discussion regarding contraception and many did not have a documented discussion regarding relationship status or sexual

Table 2 Summary of antiretroviral type and additional condom use in women using hormonal contraceptives

\begin{tabular}{llll}
\hline Participant & $\begin{array}{l}\text { Type of } \\
\text { hormonal } \\
\text { contraceptive }\end{array}$ & $\begin{array}{l}\text { Type of } \\
\text { antiretroviral }\end{array}$ & $\begin{array}{l}\text { Condoms } \\
\text { used in } \\
\text { addition }\end{array}$ \\
\hline 1 & $\begin{array}{l}\text { Long-acting } \\
\text { injection }\end{array}$ & PI & No \\
2 & IUS & NNRTI & Yes \\
3 & COCP & NNRTI & No \\
4 & COCP & None & Yes \\
5 & Long-acting & PI & No \\
6 & injection & II & Sometimes \\
7 & COCP & PI & No \\
8 & COCP & II & No \\
9 & COCP & NNRTI & No \\
10 & COCP & PI & No \\
11 & Implant & None & No \\
12 & Implant & None & No \\
13 & Implant & PI & No \\
14 & IUS & NNRTI & No \\
\hline
\end{tabular}

COCP, combined oral contraceptive pill; II, integrase inhibitor; IUS, intrauterine system; NNRTI, non-nucleoside reverse transcriptase inhibitor; $\mathrm{PI}$, protease inhibitor. activity. Understanding the importance of drug interactions between hormonal contraceptives and ARV therapy is essential to ensure that safe, effective contraception is prescribed. These discussions and discussions about future pregnancy aspirations should form a part of routine clinical care for all reproductive-aged women living with HIV, given the risk of viral transmission to partners, the importance of avoiding an unwanted pregnancy, and the potential to optimise safety of pregnancies with planning and specialist intervention.

The present study did not investigate the reasons for poor documentation of discussions regarding sexual activity, relationships and contraception; however, several hypotheses can be made. Given the retrospective nature of the study, it is unclear whether the results indicate a lack of counselling regarding sexual and reproductive health or a deficiency in documentation. In addition, it may be that women who were sexually active were more likely to initiate a discussion around this and contraception, facilitating documentation by their health care provider.

Contemporary management of HIV places significant demands on the limited time available during medical appointments. Issues including vaccination, screening for other STIs, assessment of cardiovascular and bone health, in addition to assessment of HIV disease status and adjustment of ARV therapy, may make it challenging for clinicians to address each of these topics at the recommended frequency. Previous studies have also documented the barriers doctors face in addressing sexual health in non-HIV-positive populations. ${ }^{13}{ }^{14}$ These include perceived lack of expertise or knowledge, patient reluctance, and clinician discomfort, particularly in discussing sexual health with people from minority groups including people of diverse ethnicities and sexualities. This raises the important issue of provision of specialist sexual health and contraceptive care in the clinics, and also about the important issue of enhanced clinician training in communication about both sex and contraception.

The majority of women examined during this study had a regular clinician. It is widely recognised that continuity of care and trust between treating physicians and patients are important factors in optimising the health of all patients, including those living with HIV. $^{15}$

There were no factors identified on multivariate analysis that were associated with clinician-patient discussions regarding sexual activity or contraception. This suggests that there is global inconsistency in discussions, rather than isolated settings or situations in which these discussions do or do not occur. Consequently, rates of discussions might be best increased with general guidelines such as the British HIV Association's Standards of Care for People Living with $H I V^{7}$ that suggest routine discussions and documentation of sexual activity and contraceptive use 
Table 3 Unadjusted and adjusted modelling of predictors of discussions regarding sexual activity and contraception

\begin{tabular}{|c|c|c|c|}
\hline Characteristic & Level & $\begin{array}{l}\text { Unadjusted } \\
{[\text { uRR }(95 \% \mathrm{Cl}) p]}\end{array}$ & $\begin{array}{l}\text { Adjusted } \\
{[\operatorname{aRR}(95 \% \mathrm{Cl}) p]}\end{array}$ \\
\hline \multicolumn{4}{|c|}{ Predictors of documented discussion of whether sexually active } \\
\hline Age & - & $0.97(0.95-0.99) 0.006$ & $0.99(0.97-1.01) 0.228$ \\
\hline \multirow[t]{5}{*}{ Current ARV } & $\mathrm{Pl}$ & Ref & Ref \\
\hline & NNRTI & $0.65(0.42-1.00) 0.049$ & $0.70(0.48-1.03) 0.069$ \\
\hline & $\|$ & $1.08(0.47-2.47) 0.858$ & $1.16(0.82-1.64) 0.399$ \\
\hline & Other & $0.72(0.34-1.54) 0.396$ & $0.94(0.43-2.06) 0.873$ \\
\hline & No ARV & $1.00(0.67-1.49) 0.994$ & $0.97(0.66-1.43) 0.876$ \\
\hline Previous pregnancy & - & $1.53(1.13-2.06) 0.005$ & $1.25(0.96-1.62) 0.100$ \\
\hline \multirow[t]{3}{*}{ Relationship status } & Not in relationship & Ref & Ref \\
\hline & In relationship & $1.21(0.83-1.78) 0.327$ & $1.15(0.77-1.73) 0.483$ \\
\hline & Unknown & $0.05(0.01-0.39) 0.004$ & $0.07(0.01-0.57) 0.012$ \\
\hline \multirow[t]{2}{*}{ Gender of doctor } & Female & $0.40(0.30-0.51)<0.001$ & $0.77(0.58-1.03) 0.080$ \\
\hline & Male & Ref & Ref \\
\hline Year of diagnosis (continuous) & - & $1.04(1.01-1.07) 0.027$ & $1.00(0.98-1.03) 0.740$ \\
\hline \multicolumn{4}{|c|}{ Predictors of documented discussion about contraception } \\
\hline Age & - & $0.97(0.94-1.00) 0.08$ & $1.00(0.97-1.04) 0.98$ \\
\hline \multirow[t]{5}{*}{ Current ARV } & $\mathrm{Pl}$ & Ref & \\
\hline & NNRTI & $1.10(0.56-2.15) 0.80$ & \\
\hline & $\|$ & $1.26(0.24-6.70) 0.80$ & \\
\hline & Other & $0.95(0.26-3.43) 0.93$ & \\
\hline & No ARV & $1.68(0.85-3.35) 0.14$ & \\
\hline Previous pregnancy & - & $2.00(1.18-3.38) 0.01$ & \\
\hline Documented discussion about sexual activity & - & $9.95(3.3-30.8)<0.001$ & $3.68(1.05-12.85) 0.04$ \\
\hline \multirow[t]{3}{*}{ Using contraception } & No & Ref & Ref \\
\hline & Yes & $1.53(0.84-2.78) 0.16$ & $1.78(1.00-3.19) 0.05$ \\
\hline & Unknown & $0.11(0.04-0.33)<0.001$ & $0.22(0.06-0.82) 0.024$ \\
\hline \multirow[t]{3}{*}{ Gender of doctor } & Male & Ref & Ref \\
\hline & Female & $1.51(0.66-3.50) 0.33$ & $1.19(0.80-1.78) 0.40$ \\
\hline & No regular doctor & $1.23(0.39-3.86) 0.73$ & $1.24(0.72-2.13) 0.44$ \\
\hline
\end{tabular}

aRR, adjusted risk ratio; ARV, anti retroviral; $\mathrm{Cl}$, confidence interval; II, integrase inhibitor; PI, protease inhibitor; NNRTI, non-nucleoside reverse transcriptase inhibitor; Ref, reference; uRR, unadjusted risk ratio.

annually for all patients living with HIV. These standards might be most easily met if timed with current recommendations for annual cervical cytology. ${ }^{16}$

This study demonstrated that women who used contraception were twice as likely to have had a documented discussion regarding contraception with their clinician. This may suggest that clinician-patient discussions regarding contraception influence a patient's decision to use contraception. However, this result may also reflect a clinician's documentation practices; a clinician who documents a discussion regarding contraception may be more likely to also document that the patient is using contraception.

The study is limited in that it relied on retrospective review of medical histories as evidence of clinicianpatient discussions. As identified above, documentation cannot be viewed as synonymous with actual discussions, as previous authors have demonstrated that documentation of medical consults is often incomplete. ${ }^{17}$ However, this study provides a useful insight into current practice. While prospective studies examining the same question may more accurately record discussions and contraceptive use, prospective data collection may also bias results by prompting clinicians to ask or document information regarding sexual activity and contraception. Some of our results highlight the importance of accurate and thorough documentation in medical records. Mechanisms including technology-enhanced clinical documentation and guideline implementation systems can improve documentation among health professionals and have the potential to prompt increased frequency of discussion and improve patient care. ${ }^{18}$ Importantly, this study highlights the scope for practice improvement by trialling interventions such as a standard clinical proforma, coupled with education of health care providers, to improve the frequency and documentation of discussions around sexual activity and contraception, followed by an audit to determine if the interventions have resulted in any measurable 
improvement. Further investigation to determine the applicability and utility of these mechanisms to the HIV setting is warranted.

\section{CONCLUSIONS}

Discussions between clinicians and patients regarding contraception and safer sex are important in all areas of clinical practice including for women living with HIV. Practitioner-initiated discussions may result in increased use of contraception; however, these discussions remain inconsistent. To improve the frequency of discussion we suggest combining this with the current recommendations for annual cervical cytology. Assessment of reproductive intent and need for contraception should be routinely included in these discussions. Further research should examine mechanisms to improve frequency and quality of discussions regarding sexual activity and contraception between women living with HIV and their clinicians.

Competing interests None.

Ethics approval The Alfred Health Ethics Committee.

Provenance and peer review Not commissioned; externally peer reviewed.

\section{REFERENCES}

1 Loutfy M, Raboud J, Wong J, et al. High prevalence of unintended pregnancies in HIV-positive women of reproductive age in Ontario, Canada: a retrospective study. HIV Med 2012;13:107-117.

2 Koenig L, Espinoza L, Hodge K, et al. Young, seropositive, and pregnant: epidemiologic and psychosocial perspectives on pregnant adolescents with human immunodeficiency virus infection. Am J Obstet Gynecol 2007;197(3 Suppl.):S123-S131.

3 Giles SL, Lester F. Should women with HIV, or at high risk of contracting HIV, use progestogen-containing contraception? BMJ 2013;347:f6695.

4 Delvaux T, Buve A. Hormonal contraception and HIV acquisition - what is the evidence? Eur J Contracept Reprod Health Care 2013;18:15-26.

5 World Health Organization. Hormonal Contraception and HIV. Technical Statement. 2012. http:/www.who.int/reproductivehealth/ topics/family_planning/Hormonal_contraception_and_HIV.pdf [accessed 3 February 2014].
6 Heffron R, Mugo N, Ngure K, et al. Hormonal contraceptive use and risk of HIV-1 disease progression. AIDS 2013;27:261-267.

7 British HIV Association (BHIVA). Standards of Care for People Living with HIV. 2013. http://www.bhiva.org/ standards-of-care-2012.aspx [accessed 3 February 2014].

8 Fakoya A, Lamba H, Mackie N, et al. British HIV Association, BASHH and FSRH guidelines for the management of the sexual and reproductive health of people living with HIV infection 2008. HIV Med 2008;9:681-720.

9 Bengston A, Kwok C, Salata R, et al. Hormonal contraceptive use and discontinuation among HIV-infected women in Uganda and Zimbabwe. J Acquir Immune Defic Syndr 2013;63:506-513.

10 Frost JJ, Darroch JE. Factors associated with contraceptive choice and inconsistent method use, United States, 2004. Perspect Sex Reprod Health 2008;40:94-104.

11 Matthews L, Crankshaw T, Giddy J, et al. Reproductive decision-making and periconception practices among HIV-positive men and women attending HIV services in Durban, South Africa. AIDS Behav 2013;17:461-470.

12 Department of Health, Victoria, Australia. Victorian Health Services Performance. http://performance.health.vic.gov.au/ Home.aspx [accessed 3 February 2014].

13 Gott M, Galena E, Hinchliff S, et al. "Opening a can of worms": GP and practice nurse barriers to talking about sexual health in primary care. Fam Pract 2004;21:528-536.

14 Stead ML, Brown JM, Fallowfield L, et al. Lack of communication between healthcare professionals and women with ovarian cancer about sexual issues. Br J Cancer 2003;88:666-671.

15 Hecht FM, Wilson IBI, Wu AAW, et al. Optimizing care for persons with HIV infection. Ann Intern Med 1999;131:136-143.

16 Centers for Disease Control and Prevention (CDC). Guidelines for Prevention and Treatment of Opportunistic Infections in HIV-infected Adults and Adolescents. 2009. http://www.cdc. gov/mmwr/preview/mmwrhtml/rr5804a1.htm [accessed 3 February 2014].

17 Soto C, Kleinman K, Simon S. Quality and correlates of medical record documentation in the ambulatory care setting. BMC Health Serv Res 2002;2:22.

18 Shiffman RN, Liaw Y, Brandt CA, et al. Computer-based guideline implementation systems: a systematic review of functionality and effectiveness. J Am Med Inform Assoc 1999;6:104-114. 